\title{
Anesthesia-related considerations for cerebral arteriovenous malformations
}

\author{
Tomoki Hashimoto, M.D., AND William L.Young, M.D. \\ Departments of Anesthesia and Perioperative Care, Neurosurgery, and Neurology, Center for \\ Cerebrovascular Research, University of California San Francisco, California
}

\begin{abstract}
In the optimum anesthetic management of patients with cerebral arteriovenous malformations (AVMs), the anesthesiologist should be familiar with the general pathophysiology of these lesions and various strategies for treatment. In this review, the authors outline these issues with special attention to cerebral hemodynamic changes induced by AVMs and their resection.
\end{abstract}

KEY WORDS • arteriovenous malformation • anesthesia • hemodynamics

\section{GENERAL CONSIDERATIONS IN ANESTHESIA- RELATED MANAGEMENT}

Optimum anesthetic management of patients undergoing surgical treatment of cerebral AVMs requires the following: 1) familiarity with various strategies of treatment; 2) familiarity with their general pathophysiology; and 3) the specific neuroanesthetic issues germane to this kind of treatment.

Cerebral AVMs are a complex tangle of thin-walled, abnormal vessels without an intervening capillary bed. ${ }^{23}$ The tangle of vessels, termed the "nidus," acts as an arteriovenous shunt. The malformations are presumed to be congenital lesions resulting from abnormal vascular formation during embryonic development. ${ }^{13,15,20,29,32}$ They may exert a deleterious effect on brain function via several mechanisms, including mass effects (for example, hematoma, edema, or gradually expanding abnormal vascular structures such as venous aneurysms), metabolic depression (diaschisis), and seizure activity. The most common presentation is spontaneous intracranial hemorrhage. The primary goal of treatment is to decrease the risk of spontaneous bleeding. The most conservative estimates are that $6 \%$ of patients are moderately to severely disabled after suffering a cerebral AVM hemorrhage. ${ }^{12}$ High feeding arterial pressure and venous outflow restriction are strongly associated with hemorrhagic presentation and appear to be more strongly related to initial bleeding than size, location of the lesions, or the presence of arterial aneurysms. ${ }^{8,17}$ In addition to prophylaxis against future spontaneous intracranial hemorrhage, there are certain other indications for treatment. In patients with progressive neurological deficits or intractable seizures, obliteration of high-flow feeding vessels may be of benefit, probably by treating the expanding mass effect of abnormal vascular structures.

Abbreviations used in this paper: $\mathrm{AVM}=$ arteriovenous malformation; NPPB = normal perfusion pressure breakthrough.
There are three modes of treatment for cerebral AVMs: endovascular embolization, radiosurgery, and microsurgical excision. Treatment strategies, especially for complex lesions, frequently involve more than one modality. In general, endovascular therapy is performed as a preoperative adjuvant to surgery. Using various glues or other embolic materials, the blood supply to the fistula is reduced, most commonly in several stages. This has the advantage of allowing surrounding brain regions to adapt to the circulatory changes. As a preoperative adjuvant, embolization is thought to facilitate resection by ensuring less bleeding ${ }^{40}$ and seems to be associated with improved surgery-related outcome. ${ }^{6}$ Embolization also can eliminate deep vascular pedicles that might be difficult to control surgically. The application of radiosurgery is controversial at present; it is probably ideally reserved for smaller, surgically inaccessible lesions. ${ }^{31}$

Approximately $10 \%$ of patients with cerebral AVMs also harbor intracranial aneurysms. It is of note that the converse is not true; the detection rate of cerebral AVMs in patients with aneurysms is probably similar to the detection rate of cerebral AVMs in the general population. Intracerebral hemorrhage from aneurysms is usually associated with subarachnoid hemorrhage, whereas AVMs more commonly bleed into the ventricle or into parenchyma. This explains why the occurrence of vasospasm is uncommon in cases of AVM. Spontaneous hemorrhage during the perioperative period as a result of variations in systemic blood pressure is probably less likely as well because of a "buffering" capacity of the fistula on changes in systemic pressure. ${ }^{11,38}$

\section{CEREBRAL CIRCULATORY CHANGES}

There are two primary characteristics of the cerebral circulatory changes caused by AVMs. A rapid shunt flow increases the total amount of bulk passing through the AVM. This increased flow results in cerebral arterial hypotension along the path of the shunt. Patients with cere- 
bral AVMs experience a progressive decrease in arterial pressure, which proceeds from the circle of Willis to the nidus of the AVM (Fig. 1). ${ }^{9}$ The corollary of this observation is that circulatory beds in parallel with the shunt system will be perfused at lower-than-normal pressures, even if flow remains relatively normal.

In patients with large, high-flow AVMs, there may be normal brain regions in which cerebral arterial hypotension is below the range of normal autoregulation. Despite significant cerebral arterial hypotension, a majority of patients do not experience ischemic symptoms. Hypotensive normal brain regions can be demonstrated to have normal rates of tissue perfusion, implying the existence of some adaptive change in total cerebrovascular resistance.

This phenomenon may be explained by adaptive autoregulatory displacement. ${ }^{16}$ In vascular territories adjacent to cerebral AVMs, the lower limit of the autoregulation curve appears to be shifted to the left, which is in contrast to the effect of chronic systemic (essential) arterial hypertension on the cerebral autoregulation curve. ${ }^{41}$ This adaptive shift to the left places the lower limit at a level considerably lower than that postulated for normal brain $(50$ or $60 \mathrm{~mm} \mathrm{Hg}) .{ }^{26,34}$ Therefore, the presence of chronic hypotension does not necessarily result in vasoparalysis in the arteriolar resistance bed. There is generally a preserved responsiveness to $\mathrm{CO}_{2}$ pre- and postresection, which lends further support to the notion of intact autoregulatory capacity. ${ }^{41}$

Historically, it has been believed that it is not the AVM itself but rather decreased perfusion pressure in adjacent, functional tissue that is responsible for both pretreatment ischemic and posttreatment hyperemic symptoms - namely so-called "cerebral steal" and "normal perfusion pressure breakthrough." These widely discussed concepts have limited anecdotal evidence to support their existence.

So-called cerebral steal has been suggested as an explanation for focal neurological deficits in patients with cerebral AVM; the concept of cerebral steal is assumed to be attributable, by inference, to local hypotension and hypoperfusion. It has been postulated that arteriolar vascular resistance in territories adjacent to AVMs is at or near a state of maximum vasodilation, and therefore cerebral steal ensues if perfusion pressure decreases. Although cerebral arterial hypotension in normal brain areas is common in AVMs patients, it is rare (10\% of cases) for focal neurological deficits to manifest clinically. Moreover, there is no relation between local hypotension and focal neurological deficits. ${ }^{25}$ It is likely that local mass effects produced by the abnormal vessels of the AVMs are more important than local "hemodynamic failure" to account for symptomatic focal neurological deficits unrelated to intracerebral hemorrhage or seizure activity.

The intraoperative appearance of the operative site-related diffuse bleeding or brain swelling and the postoperative occurrence of hemorrhage or swelling has been attributed to NPPB or hyperemic complications. There are difficulties in studying NPPB-type complication. First, there appears to be a very heterogeneous set of criteria used by different authors. Second, the postoperative incidence of this type of complication is probably lower than $5 \% .{ }^{37}$ The increase in global cerebral blood flow after resection of a cerebral AVM appears to be associated with NPPB-type complications, but there is no relationship with preoperative regional arterial hypotension. ${ }^{37}$ Normal perfusion pressure breakthrough is attributed to cerebral hyperemia caused by repressurization of previously hypotensive regions. This theory assumes that chronic dilation of vessels in hypotensive/ischemic territory leads to a loss of autoregulation. ${ }^{34}$ There are, however, several observations that contradict this theory. First, cerebral hyperemia that develops after resection of an AVM is global and not limited to ipsilateral side of the AVM.${ }^{37}$ Second, there is no relationship between cerebral blood flow changes after resection and the degree of arterial hypotension induced by AVM shunts. ${ }^{37}$ Third, in hypotensive regions, autoregulatory response is not impaired but is intact and

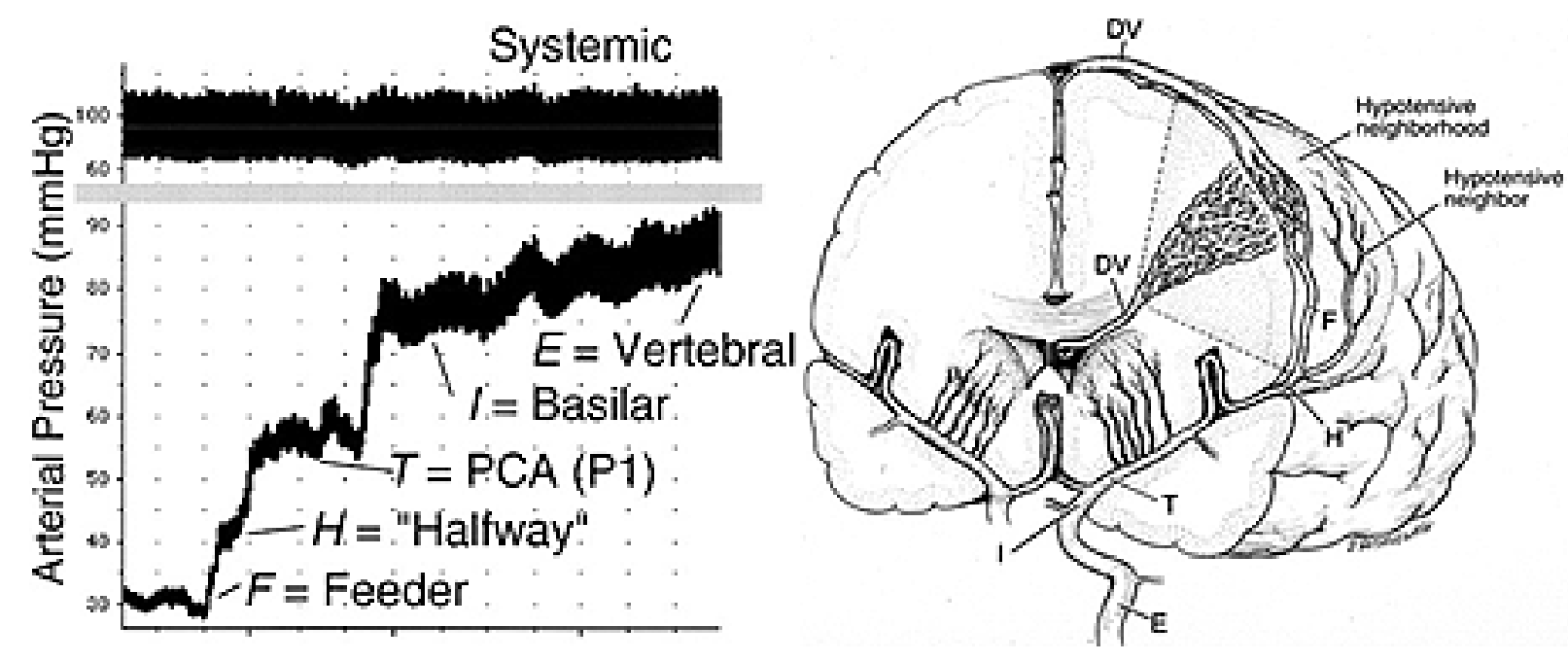

Fig. 1. Left: Graph showing a representative pressure recording obtained using a microcatheter. $\mathrm{F}=$ feeding artery; $\mathrm{H}=$ halfway between feeding artery and posterior cerebral artery; $\mathrm{T}=$ posterior cerebral artery; $\mathrm{I}=$ basilar artery; $\mathrm{E}=$ vertebral artery. Right: Schematic view illustration showing vessels corresponding to those at left. DV $=$ draining vein. 
shifted to the left. ${ }^{39,41}$ Finally, cerebrovascular reactivity to $\mathrm{CO}_{2}$ is preserved after AVM resection, ${ }^{42}$ suggesting that the vessels in previously hypotensive territories are not paralyzed.

Although the states of so-called cerebral steal and NPPB probably do exist in some rare minority of cases, if they exist, they are the exception rather than the rule. With regard to perioperative management, the diagnosis of NPPB should be one of exclusion after all other correctable causes for malignant brain swelling or bleeding have been excluded. In addition to other supportive and resuscitative measures, the prevention of severe postoperative hypertension may be useful in preventing and treating this syndrome.

\section{ANESTHETIC MANAGEMENT Preoperative Management}

Because the resection of cerebral AVMs is rarely performed as an emergency procedure, a careful review of the patient's perioperative status and assessment of potential intraoperative difficulties should be possible. Preexisting medical conditions should be optimized. The existence of neurological dysfunction, as a result of presenting hemorrhage, presumed effect of the AVM, or preoperative embolization (infarction or edema), must be considered in such treatment-related choices as monitoring, vascular access, anesthetic agents, vasoactive drugs, muscle relaxants, and postoperative airway control. A critical consideration throughout the operative period is the potential for massive and rapid blood loss. This choice of intraoperative monitoring is tempered by this eventuality. Adequate blood products, along with access for their administration, should be at hand.

\section{Monitoring}

In addition to routine monitors - such as electrocardiography, pulse oximeter, end-tidal $\mathrm{CO}_{2}$, temperature probe, and direct arterial pressure transduction-central access should be considered for resection of larger lesions. It is not always possible to predict preoperatively which patient will subsequently require extraordinary levels of induced hypotension or aggressive volume therapy. Such cases are a minority, but central venous or pulmonary artery catheters may be useful for management of complicated cases.

Unfortunately, our ability to monitor the central nervous system lags far behind our ability to monitor other systems, and the existence of suitable technologies is still in its infancy. There is no consensus about optimum monitoring techniques, primarily because there is a dearth of commercially available technologies.

Transduction of vascular pressures in the operative field may aid the surgeon in differentiating arterial and venous structures. In certain cases, it may assist in the decision of whether a draining vein that interferes with surgical access to the nidus can be sacrificed. Proximal arterial pressure is measured during a temporary occlusion of the vein; if the pressure does not change, this implies that alternate venous pathways are sufficient to prevent distention of the nidus and rupture. Technically, direct puncture of feeding arteries with 26-guage needles and draining veins in the operative field is a simple procedure that poses little, if any, risk. Measurements of vascular pressure have been reported by several groups.

\section{Anesthetic Technique}

Choice of Agents. Intracranial pressure control is rarely a problem in the patient with an AVM in whom elective resection is being performed. Nevertheless, because decreased intracranial compliance may be present, the usual caveats about avoiding cerebral vasodilators are reasonable.

Except for cerebral vasodilators, the specific choice of an anesthestic may be guided primarily by other coexisting conditions. An isoflurane/ $\mathrm{N}_{2} \mathrm{O}$ technique offers good systemic blood pressure control. The use of total intravenous anesthetic methods or combinations of inhalational and intravenous methods may optimize rapid emergence from the state of anesthesia. ${ }^{27}$ In some centers additional barbiturate loading is used during the resection to afford additional protection against cerebral ischemia, resulting in perhaps a greater degree of brain relaxation and protection against acute hyperemia. ${ }^{33}$ Barbiturates can be titrated to an electroencephalography endpoint of burst suppression. The main drawback of barbiturate use is delayed emergence from an anesthetized state, thus forgoing an early postoperative neurological exam. There is no evidence that outcome is affected. If metabolic suppression is desired (and we by no means endorse this as a unique, effective method of intraoperative protection ${ }^{35}$ ), propofol or etomidate may be considered. The use of metabolic suppression may be useful in the event of an intraoperative catastrophe (see Induced Hypotension).

Nonpharmacological Cerebral Protection. The goals of modern neuroanesthesia should not revolve around provision of pharmacological brain protective therapy, per se. There are a number of basic considerations that will maximize nonpharmacological cerebral protection and provide protection from injury (Table 1). There are two general types of damage that protective efforts are undertaken to prevent: neurosurgical (anatomical) and anesthetic (physiological) trespass. Possible mechanisms of neurosurgeon-induced injury include brain retraction, direct vascular injury (ischemia, thrombosis, and venous occlusion), and mechanical disruption of neuronal tissue or white matter tracts. Anesthesia-induced injury may result from systemic hypo- or hypertension, decreased $\mathrm{O}_{2}$ content, hypoosmolarity, or hyperglycemia. It must be stressed that mechanisms of damage are interactive. Modest amounts of brain retraction coupled with modest reduction of systemic blood pressure, for example, may have synergistic effects on patient emergence or on neurological outcome.

Management goals should include ensuring brain relaxation, controlled systemic and cerebral hemodynamics, maintenance of isotonicity and euglycemia, mild hypothermia, and a controlled emergence from the state of anesthesia.

\section{Brain Relaxation}

The process of ensuring adequate brain relaxation begins with proper positioning of the patient's head to promote intracranial venous drainage. The least amount of flexion and rotation necessary for the operative approach 
TABLE 1

Summary of considerations in nonpharmacologic brain protection

relaxed brain

good head position

cerebrospinal fluid drainage

diuretics/osmotherapy

avoid excessive cerebral vasodilators

modest hypocapnia

controlled systemic \& cerebral hemodynamics euvolemia

optimal cerebral perfusion pressure

fluid \& electrolyte management

isotonicity

euglycemia

temperature management

toleration of intraop modest hypothermia

prevention of postop hyperthermia

controlled emergence

tailored awakening

autonomic control

should be planned in conjunction with the surgeon. Careful positioning of the head may also prevent postoperative tongue swelling, a rare but morbid occurrence. One rule of thumb might be that there should be two-finger breadths per $70 \mathrm{~kg}$ between the mandible and clavicle (not the sternum) after the head is positioned in rigid pin fixation. The head of the table should be positioned to prevent venous engorgement.

Cerebral spinal fluid removal is an effective means of inducing brain relaxation and is obtained by direct lumbar puncture or ventricular drainage. Diuretic therapy with mannitol and/or furosemide is widely applied. The most important consideration for the choice of an intraoperative anesthetic is the avoidance of cerebral vasodilators. Modest hypocapnia should be applied sparingly as an adjuvant to brain relaxation, but levels below $30 \mathrm{~mm} \mathrm{Hg}$ should have a specific indication.

\section{Controlled Systemic and Cerebral Hemodynamics}

Euvolemia and Blood Pressure Control. Fluid restriction has been a time-honored means of guarding against brain swelling in the patient undergoing a neurosurgical procedure. Adequate volume status to maintain stable systemic hemodynamics, especially with the application of induced hypotension, may require liberal fluid administration. Recent evidence reconciles these two apparently divergent goals (the influence of serum tonicity on fluid movement into the brain in Fluid and Electrolyte Management).

Control of cerebral hemodynamics begins with control of systemic arterial pressure, which in turn is predicated on adequate cardiac preload (euvolemia). Iatrogenic dehydration, as practiced in years past, has no place in modern neurosurgical practice. Indeed, in the setting of aneurysmal subarachnoid hemorrhage, it is clearly deleterious. During manipulation of the intracranial contents or their vascular supply, the anesthesiologist should strive to maintain the optimal cerebral perfusion pressure - that is, the highest clinically acceptable blood pressure in the given clinical circumstance. Brain relaxation is probably also served by maintenance of a normal arterial pressure.
Cerebral blood volume is kept to a minimum by appropriate autoregulatory vasoconstriction. ${ }^{28}$

Induced Hypotension. In contrast to the current trend to maintain normotension during intracranial aneurysm clipping, induced hypotension is frequently useful during the resection of a cerebral AVM. This is especially germane to large AVMs in which the arterial supply is deep. Bleeding from these small, deep feeding vessels may be difficult to control, and decreasing the arterial pressure facilitates surgical hemostasis. The subject of induced hypotension is discussed extensively in the neuroanesthesia literature.

During uncontrolled bleeding, the surgeons may be forced to place clips blindly in an attempt to stem the hemorrhage. In this event, barbiturate therapy may be indicated, and it could be used as a means of or as an adjuvant to the induction of mild or moderate temporary arterial blood pressure reduction until bleeding is controlled. Induction of systemic hypotension with a pure vasodilator has theoretical disadvantages. In the setting of emergency intracranial vascular occlusion to control hemorrhage, the distal perfusion field of the occluded artery will have little or no opportunity to recruit collateral flow from neighboring (relatively vasodilated) normal arterial supply regions. The clinician, however, should use whatever measures with which he or she is comfortable and adept to reduce blood pressure expeditiously as demanded by the clinical situation.

\section{Fluid and Electrolyte Management}

There is a convincing body of evidence that serum osmolality strongly influences water movement into both the normal and damaged brain. ${ }^{43}$ Even mildly hypotonic fluids such as lactated Ringer solution, if given in sufficient quantity, may aggravate brain swelling more than isotonic crystalloids or colloids. Isotonic fluid replacement in which blood, saline, or hetastarch is used after forebrain ischemia in the rat appears to yield similar results in terms of the formation of cerebral edema. ${ }^{36}$ The most important point is that fluid should never be withheld at the expense of a stable cardiovascular status. Serum osmolarity can be easily monitored if large volumes of crystalloid are needed. The issue of how colloid oncotic pressure affects the formation of brain edema remains a controversial topic. ${ }^{7,18,19}$ A recent experimental study indicates that a reduction of colloid oncotic pressure without decreased osmolality may exacerbate posttraumatic brain edema. ${ }^{7}$ Aggressive administration of isotonic crystalloids may worsen brain edema by decreasing colloid oncotic pressure. $^{7}$

There is considerable evidence that glucose aggravates cerebral injury not only in animals but also in humans. ${ }^{21,22}$ Routine perioperative steroid administration may cause some degree of hyperglycemia. In the absence of clear guidelines, the most rational approach is to avoid glucosecontaining fluids unless there is a specific indication. One such indication would be a case in which a diabetic patient receives insulin therapy. In this case, "tight" rather than "loose" control of serum glucose seems reasonable; it is probably not worth the risk of causing hypoglycemia in an anesthetized patient for any presumptive protective effect of lowering a mildly elevated glucose level.

How does one define optimum levels? Observational evidence suggests that elevations in glucose levels are 
associated with worsened outcome after various types of brain injury. For example, in severely head injured patients (with Glasgow Coma Scale scores $\leq 8$ ), a postoperative serum glucose level greater than $200 \mathrm{mg} / \mathrm{dl}$ was associated with a worsened outcome. ${ }^{21}$

\section{Toleration of Modest Hypothermia}

Mild hypothermia (in which the core temperature decreases are as little as $1.5-3^{\circ} \mathrm{C}$ ) has been shown to confer dramatic cerebral protection against ischemic insult in animal models. ${ }^{3}$ This protective effect is greater than what would be expected from metabolic suppression alone and may be related to a decrease in excitatory neurotransmitter release from ischemic cells. ${ }^{2}$ Although the authors of two randomized controlled clinical trials showed a favorable result of mild hypothermia in patients with severe traumatic brain injury, ${ }^{5,24}$ the efficacy of prophylactic mild hypothermia during craniotomy has not yet been validated. Hypothermia appears to play a more significant role in determining outcome from ischemic insult than the choice of anesthetic agents. ${ }^{30}$

Recent investigations have revealed that anesthetized patients can be easily cooled to the cerebroprotective range $\left(33-34^{\circ} \mathrm{C}\right)$, although complete intraoperative rewarming may be difficult to achieve. ${ }^{1}$ Even mild degrees of hypothermia are not without potential risk. Passive rewarming is associated with peripheral vasoconstriction, shivering, and subsequent increases in oxygen consumption and myocardial work. Drug metabolism is decreased, prolonging the effect of even short-acting anesthetic agents. Postoperative hypothermia $\left(<35^{\circ} \mathrm{C}\right)$ is complicated by increased rates of myocardial ischemia, angina, and arterial hypoxemia in populations at risk for coronary artery disease. ${ }^{10}$ Moderate hypothermia $\left(<33^{\circ} \mathrm{C}\right)$ has other well-documented potential effects, including increased susceptibility to infection, cardiac arrhythmias, hypercoagulability, thrombocytopenia, impaired platelet aggregation, and activation of fibrinolysis, all of which reverse with rewarming. ${ }^{1,10}$ Most of these adverse effects have been observed in patients who are moved from the operating room while still hypothermic. It is unclear whether the potential benefits of cerebral protection gained from mild hypothermia and partial rewarming are offset by the systemic physiological stress induced, particularly if shivering occurs upon waking.

Rather than struggling to maintain normothermia, a conservative recommendation would be to achieve mild body temperature reduction $\left(34-35^{\circ} \mathrm{C}\right)$ until surgical closure is imminent, and only then should active rewarming with water blankets and convective devices begin. Careful temperature monitoring should continue throughout the perioperative period; hyperthermia must be avoided as it potentiates ischemic damage. ${ }^{4}$

As of this writing, there is currently a multicenter randomized trial planned in which the participants will evaluate the efficacy of intraoperative hypothermia to improve outcome after intracranial aneurysm clipping; preliminary data have been presented. ${ }^{14}$

\section{PERIOPERATIVE CARE}

\section{Emergence and Initial Recovery: Blood Pressure Control}

A particularly challenging aspect of perioperative care is emergence and initial recovery from the state of anes- thesia. A moderate phenylephrine-induced blood pressure augmentation (20-30\% above normal mean arterial pressure) may be needed during drying of the operative bed to inspect for hemostasis. After hemostasis is achieved and the volatile agent is discontinued, antihypertensive agents such as labetalol or esmolol can be used to maintain the patient's blood pressure within $10 \%$ below the baseline values.

We would emphasize that, without firm outcome data indicating the superiority of one drug regimen or another, the choice of agent to manipulate blood pressure must be placed in the context of the clinical situation (for example, avoiding beta adrenergic blockers in case of bronchospastic airway disease or administration of nitroglycerin in cases of coronary artery disease) and the experience of the practitioner.

The most sensitive index of cerebral function remains the neurological examination. A prompt emergence from anesthesia will ensure that drug residua are not confused with obscure focal neurological damage. Control of systemic hemodynamics is of critical importance during the emergence phase as the patient makes the transition from the anesthetized to the conscious state.

\section{POSTOPERATIVE MANAGEMENT}

Figuratively speaking, the sword of aggressive blood pressure control can cut both ways. There are cases of ischemic deficits caused, for example, by the intraoperative sacrifice of an en passage feeding vessel (a vessel feeding the AVM and also sending distal branches to normal brain) that may result in a deficit ascribed to brain retraction or the resection itself. Marginally perfused areas may be critically dependent on collateral perfusion pressure. The maintenance of low or even normal blood pressure may be inadequate, if unrecognized, and result in infarction. Unfortunately, the only reliable means of verifying borderline perfusion states at the present time is intraoperative or immediate postoperative angiography.

Postoperative hyperthermia may be detrimental ${ }^{4}$ and even exacerbated by intraoperative mild induced hypothermia. ${ }^{1}$ Therefore, careful attention should be paid to a patient's temperature during the postoperative period.

\section{References}

1. Baker KZ, Young WL, Stone JG, et al: Deliberate mild intraoperative hypothermia for craniotomy. Anesthesiology 81: 361-367, 1994

2. Busto R, Dietrich WD, Globus MY, et al: The importance of brain temperature in cerebral ischemic injury. Stroke 20: 1113-1114, 1989

3. Busto R, Dietrich WD, Globus MY, et al: Small differences in intraischemic brain temperature critically determine the extent of ischemic neuronal injury. J Cereb Blood Flow Metab 7: 729-738, 1987

4. Chen H, Chopp M, Welch KM: Effect of mild hyperthermia on the ischemic infarct volume after middle cerebral artery occlusion in the rat. Neurology 41:1133-1135, 1991

5. Clifton GL, Allen S, Barrodale P, et al: A phase II study of moderate hypothermia in severe brain injury. J Neurotrauma 10: 263-273, 1993

6. DeMeritt JS, Pile-Spellman J, Mast H, et al: Outcome analysis of preoperative embolization with N-butyl cyanoacrylate in cerebral arteriovenous malformations. AJNR 16:1801-1807, 1995

7. Drummond JC, Patel PM, Cole DJ, et al: The effect of the re- 
duction of colloid oncotic pressure, with and without reduction of osmolality, on post-traumatic cerebral edema. Anesthesiology 88:993-1002, 1998

8. Duong DH, Young WL, Vang MC, et al: Feeding artery pressure and venous drainage pattern are primary determinants of hemorrhage from cerebral arteriovenous malformations. Stroke 29:1167-1176, 1998

9. Fogarty-Mack P, Pile-Spellman J, Hacein-Bey L, et al: The effect of arteriovenous malformations on the distribution of intracerebral arterial pressures. AJNR 17:1443-1449, 1996

10. Frank SM, Beattie C, Christopherson R, et al: Unintentional hypothermia is associated with postoperative myocardial ischemia. The Perioperative Ischemia Randomized Anesthesia Trial Study Group. Anesthesiology 78:468-476, 1993

11. Gao E, Young WL, Pile-Spellman J, et al: Cerebral arteriovenous malformation feeding artery aneurysms: a theoretical model of intravascular pressure changes after treatment. Neurosurgery 41:1345-1358, 1997

12. Hartmann A, Mast H, Mohr JP, et al: Morbidity of intracranial hemorrhage in patients with cerebral arteriovenous malformation. Stroke 29:931-934, 1998

13. Hashimoto T, Lam T, Boudreau NJ, et al: Abnormal balance in the angiopoietin-Tie2 system in human brain arteriovenous malformations. Circ Res 89:111-113, 2001

14. Hindman BJ, Todd MM, Gelb AW, et al: Mild hypothermia as a protective therapy during intracranial aneurysm surgery: a randomized prospective pilot trial. Neurosurgery 44:23-33, 1999

15. Kader A, Goodrich JT, Sonstein WJ, et al: Recurrent cerebral arteriovenous malformations after negative postoperative angiograms. J Neurosurg 85:14-18, 1996

16. Kader A, Young WL: The effects of intracranial arteriovenous malformations on cerebral hemodynamics. Neurosurg Clin $\mathbf{N}$ Am 7:767-781, 1996

17. Kader A, Young WL, Pile-Spellman J, et al: The influence of hemodynamic and anatomic factors on hemorrhage from cerebral arteriovenous malformations. Neurosurgery 34:801-808, 1994

18. Kaieda R, Todd MM, Cook LN, et al: Acute effects of changing plasma osmolality and colloid oncotic pressure on the formation of brain edema after cryogenic injury. Neurosurgery 24:671-678, 1989

19. Kaieda R, Todd MM, Warner DS: Prolonged reduction in colloid oncotic pressure does not increase brain edema following cryogenic injury in rabbits. Anesthesiology 71:554-560, 1989

20. Kilic T, Pamir MN, Kullu S, et al: Expression of structural proteins and angiogenic factors in cerebrovascular anomalies. Neurosurgery 46:1179-1192, 2000

21. Lam AM, Winn HR, Cullen BF, et al: Hyperglycemia and neurological outcome in patients with head injury. J Neurosurg 75:545-551, 1991

22. Lanier WL: Glucose management during cardiopulmonary bypass: cardiovascular and neurologic implications. Anesth Analg 72:423-427, 1991

23. Mandybur TI, Nazek M: Cerebral arteriovenous malformations. A detailed morphological and immunohistochemical study using actin. Arch Pathol Lab Med 114:970-973, 1990

24. Marion DW, Penrod LE, Kelsey SF, et al: Treatment of traumatic brain injury with moderate hypothermia. N Engl J Med 336:540-546, 1997

25. Mast H, Mohr JP, Osipov A, et al: 'Steal' is an unestablished mechanism for the clinical presentation of cerebral arteriovenous malformations. Stroke 26:1215-1220, 1995

26. Nornes H, Grip A: Hemodynamic aspects of cerebral arteriovenous malformations. J Neurosurg 53:456-464, 1980

27. Ravussin P, Tempelhoff R, Modica PA, et al: Propofol vs. thiopental-isoflurane for neurosurgical anesthesia: comparison of hemodynamics, CSF pressure, and recovery. J Neurosurg Anesth 3:85-95, 1991
28. Rosner MJ: Cerebral perfusion pressure: link between intracranial pressure and systemic circulation, in Wood JH (ed): Cerebral Blood Flow: Physiologic and Clinical Aspects. New York: McGraw-Hill, 1987, pp 425-448

29. Rothbart D, Awad IA, Lee J, et al: Expression of angiogenic factors and stuctural proteins in central nervous system vascular malformations. Neurosurgery 38:915-925, 1996

30. Sano T, Drummond JC, Patel PM, et al: A comparison of the cerebral protective effects of isoflurane and mild hypothermia in a model of incomplete forebrain ischemia in the rat. Anesthesiology 76:221-228, 1992

31. Sisti MB, Kader A, Stein BM: Microsurgery for 67 intracranial arteriovenous malformations less than $3 \mathrm{~cm}$ in diameter. J Neurosurg 79:653-660, 1993

32. Sonstein WJ, Kader A, Michelsen WJ, et al: Expression of vascular endothelial growth factor in pediatric and adult cerebral arteriovenous malformations: an immunocytochemical study. J Neurosurg 85:838-845, 1996

33. Spetzler RF, Martin NA, Carter LP, et al: Surgical management of large AVM's by staged embolization and operative excision. J Neurosurg 67:17-28, 1987

34. Spetzler RF, Wilson CB, Weinstein P, et al: Normal perfusion pressure breakthrough theory. Clin Neurosurg 25:651-672, 1978

35. Todd MM, Warner DS: A comfortable hypothesis reevaluated. Cerebral metabolic depression and brain protection during ischemia. Anesthesiology 76:161-164, 1992

36. Warner DS, Boehland LA: Effects of iso-osmolal intravenous fluid therapy on post-ischemic brain water content in the rat. Anesthesiology 68:86-91, 1988

37. Young WL, Kader A, Ornstein E, et al: Cerebral hyperemia after arteriovenous malformation resection is related to "breakthrough" complications but not to feeding artery pressure. The Columbia University Arteriovenous Malformation Study Project. Neurosurgery 38:1085-1095, 1996

38. Young WL, Kader A, Pile-Spellman J, et al: Arteriovenous malformation draining vein physiology and determinants of transnidal pressure gradients. The Columbia University AVM Study Project. Neurosurgery 35:389-396, 1994

39. Young WL, Kader A, Prohovnik I, et al: Pressure autoregulation is intact after arteriovenous malformation resection. Neurosurgery 32:491-497, 1993

40. Young WL, Pile-Spellman J: Anesthetic considerations for interventional neuroradiology. Anesthesiology 80:427-456, 1994

41. Young WL, Pile-Spellman J, Prohovnik I, et al: Evidence for adaptive autoregulatory displacement in hypotensive cortical territories adjacent to arteriovenous malformations. Columbia University AVM Study Project. Neurosurgery 34:601-611, 1994

42. Young WL, Prohovnik I, Ornstein E, et al: The effect of arteriovenous malformation resection on cerebrovascular reactivity to carbon dioxide. Neurosurgery 27:257-267, 1990

43. Zornow MH, Todd MM, Moore SS: The acute cerebral effects of changes in plasma osmolality and oncotic pressure. Anesthesiology 67:936-941, 1987

Manuscript received October 22, 2001.

Accepted in final form October 31, 2001.

Portions of this work were supported by Grants Nos. K24 NS0209 and RO1 NS27713.

Address reprint requests to: William L.Young, M.D., Center for Cerebrovascular Research, University of California, San Francisco, 1001 Potrero Avenue, \#3C-38, San Francisco, California 94110. email: CCR@anesthesia.ucsf.edu. 